International Journal on Cybernetics \& Informatics (IJCI) Vol. 5, No. 2, April 2016

\title{
COMPACT MICROWAVE PLANAR BAND PASS FILTER
}

\author{
Ambily $\mathrm{K}^{1}$ and Anila P V \\ ${ }^{1}$ Dept of Electronics and Communication, Mar Athanasius Engineering College \\ A.P.J Abdul Kalam Technological University, Kerala, India \\ ${ }^{2}$ Assistant Professor, Dept of Electronics and Communication Engineering \\ Mar Athanasius Engineering College, Kothamangalam Kerala, India
}

\begin{abstract}
The need for the compactness of microwave filter at high frequency application has lead to the design of microwave planar band pass filter which passes certain range of frequencies. The aim of this paper is to study and implement a compact microwave planar band pass filter operates in $3.1 \mathrm{GHz}-10.6 \mathrm{GHz}$ which is the ultra-wide band range. It uses multiple mode resonator (MMR) and inter digital coupled lines to improve upper stop band performance and coupling degree. Simulation of the proposed microwave filter structures is done with the help of HFSS (High Frequency Structure Simulator) software.
\end{abstract}

\section{KEYWORDS}

MMR, SLFSIR, resonator

\section{INTRODUCTION}

The increasing scale of modern wireless communication application and radar system in today's technology has boosted the demand for microwave filters as they are playing an essential role in Transmit-Receive system.

In circuit theory, a filter is an electrical network that alters the phase and/or amplitude characteristics of a signal with respect to frequency. A filter will not add new frequencies to the input signal, nor will it change the component frequencies of the signal, but it will change the relative amplitudes of the various frequency components and their phase relationships. Filters are used in electronic systems to emphasize signals in certain frequency ranges and reject signals in other frequency ranges. A microwave filter is a two-port network used to control the frequency response at a certain point in a microwave system by allowing transmission at frequencies within the pass band of the filter and attenuation in the stop band of the filter. Typical frequency responses include high-pass, low-pass, band reject, and band-pass characteristics. Applications can be found in virtually any type of test and measurement system, microwave communication and radar.

A band pass filter allows transmission of a limited band of frequencies and rejects all other frequencies below or above frequency band. It has parallel tuned circuit in the shunt arm and

DOI: $10.5121 /$ ijci.2016.5231 
series tuned circuit in series arm . Here a microwave planar band pass filter in ultra wide band range (3.1-10.6) GHz with circular stepped impedance stubs is implemented with a low insertion loss in pass band. For microwave range higher than $500 \mathrm{MHz}$ the passive filters are mostly realized by using either planar transmission lines, or waveguides. Although waveguide components have low losses, and can handle higher power than the planar transmission lines it is not preferable in new communications systems that require the mobility, because of the their large size and heavy weight. Moreover, fabrication processes of the waveguide components are more expensive than that for planar transmission lines. Therefore most of the microwave filters are designed using the transmission lines. Variety of structures such as stepped impedance stubs, coupled lines, single mode resonator, multi mode resonators and coupled resonators can be used for implementation.

\section{METHODS IN FILTER DESIGN}

\subsection{COMPACT UWB BAND PASS FILTER WITH MMR}

The MMR used for this filter design consists of one half-wavelength $\lambda / 2$ low impedance line section in the center and two identical $\lambda / 4$ high-impedance line sections at the two sides. Here first three resonant frequencies of the MMR are properly adjusted to be placed quasi equally within the UWB. After that, the parallel-coupled lines at the two sides are longitudinally stretched to raise the frequency dispersive coupling degree with the coupling peak near the center of the UWB.

It was initially designed in [1] such that that the first two resonant modes of the MMR is utilized together with the input/output parallel-coupled lines to achieve a wide pass band with four transmission poles. Another modification of the filter is presented in [2],[3] by making first three resonant modes of the MMR constructed to realize five transmission poles with lowered return loss in the whole pass band range. Following the works in [1],[2] and [3] the MMR here is to be properly modified in configuration so as to reallocate its first three resonant modes close to the lower-end, center, and upper-end of the targeted UWB pass band. By forming a MMR and introducing quarter-wavelength parallel coupled lines in the input and output ports, a UWB pass band with five transmission poles is achieved and it is finalized as the result.

\subsection{FILTERS WITH STUB LOADED MMR}

UWB band pass filter using MMR of stepped-impedance configuration was initially reported in [4]. But these stepped-impedance MMR-based filter suffered from a high insertion loss, narrow upper stop band as well as worse selectivity.

A new approach was the design of filter based on electromagnetic bandgap (EBG) embedded MMR which is reported in [5] to widen the upper stop band. The core piece of these two filters is a stub-loaded multiple-mode resonator (MMR). The MMR is constructed by loading three open stubs in a uniform-impedance resonator that means and two uniform-impedance stubs of lengths $1_{\mathrm{s}}$ at the symmetrical side locations and one stepped-impedance stub at the center of length $1_{\mathrm{c}}$. Five modes, includingthree even modes and two odd modes, could be designed within UWB band .The odd and even resonances of an unloaded MMR occur alternatively. All resonances move down to lower frequencies as $1_{\mathrm{s}}$ is increased and this implies circuit miniaturization. Only the 
even modes move down to lower frequencies when $1_{c}$ is increased. The two odd modes could be located within the UWB band by properly designing the horizontal impedance resonator and the side stubs and the even modes could be flexibly tuned while the odd modes are fixed. The two transmission zeros generated by the stepped-impedance stub are at the lower and upper cut-off frequencies, resulting in a sharp pass band performance. These results demonstrate that this UWB filters has much better electrical performances of UWB pass band than the filters mentioned in [4] and [5]. The filters explained in papers [1-5] had the disadvantages like narrow upper-stop owing to the appearance of parasitic pass bands, electrically large in size which is impractical in the implementation of hand-held UWB devices and the tight line spacing between parallel coupled lines for strong coupling is hard for fabrication.

\subsection{FILTER WITH SLFSIR}

Q X Chu etal came up with a more compact UWB band-pass filter using a stub loaded folded stepped impedance resonator (SLFSIR) and aperture-backed inter digital coupled lines .This filter comes up with a solution to all problems discussed in [1-5]. Several prototype UWB filters have been reportedly developed based on principles, such as dual-stop band features [6] and pseudointer digital structure [7]. The SLFSIR is designed such that the first four resonant frequencies of it are properly adjusted to be placed evenly within the UWB while the fifth resonant frequency is made above $16.0 \mathrm{GHz}$ to improve upper stop band performance. The aperture-backed inter digital coupled lines can create a transmission zero to eradicate the fifth resonant mode of the SLFSIR to make a wider upper-stop band. To make a wide pass band covering the whole UWB band, the aperture width and parallel-coupled lines length should be suitably chosen to get proper coupling [8]. The inter-digital coupled lines can increase the coupling degree to relax the tight line spacing. The two side stubs with varied lengths in SLFSIR can provide an extra degree of freedom for adjusting the locations of the first four resonant frequencies in an alternative way. The filter proposed by them has some attractive features like a wide and deep upper-stop band with insertion loss higher than $20 \mathrm{~dB}$ in the $11.7-19.0 \mathrm{GHz}$ range, relaxed tight line spacing and miniaturized size. These MMR based UWB filters are still limited by the existence of periodic and narrow pass bands in the upper-stop band.

\section{PROPOSED FILTER}

The compact UWB band pass filter uses MMR which is made by uniting three pairs of circular impedance stepped stubs in shunt to a high impedance micro-strip configuration, as shown in figure 1. A band-pass filter can be constructed using any units that can resonate. Filters using stubs can clearly be made band-pass. Stub is the common component of distributed filters. Over a narrow range of frequencies, a stub can be used as an inductor or a capacitor (its impedance is determined by its length) but over a wide band it act as a resonator. This compact UWB band pass filter uses stepped stubs. It can easily implement steps in characteristic impedance of the configuration, which introduces a discontinuity in the transmission. 


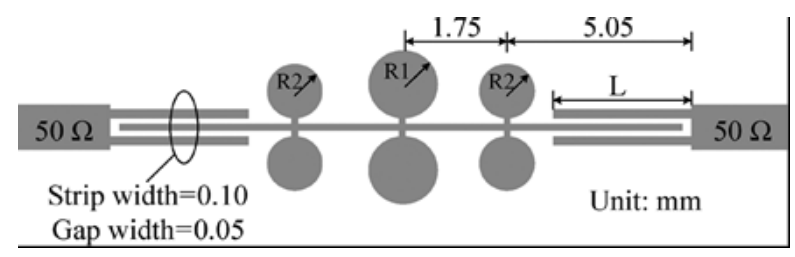

Figure 1: Filter structure

\section{STRUCTURAL DESCRIPTION}

Resonators are fundamental elements which usually determine the size of the filter. The effective approach for miniaturizing the filter size is to reduce the resonator size. This can be achieved by two methods. One approach is to modify the physical structures another approach is to modify the traditional resonators to generate additional modes, which makes the resonator to behave as an MMR.

Multiple mode resonator resembles to a metal box of fixed dimensions that can support a number of resonant modes at a given frequency. They combine different multiple modes which constitute narrow bands into a wide band. That is multiple mode resonator has multiple resonance behaviour in its pass band.

The compact UWB band pass filter uses MMR which is formed by attaching three pairs of circular impedance-stepped stubs in shunt to a high impedance micro-strip line. A band-pass filter can be constructed using any units that can resonate. Filters using stubs can clearly be made bandpass. Stub is the common component of distributed filters.Short-circuit, nominally quarterwavelength stubs behave as shunt LC resonator and an open-circuit nominally quarterwavelength stub behaves as a series LC resonator.

The compact UWB band pass filter uses stepped stubs. It can easily implement steps in characteristic impedance of the configuration, which paves the way for a discontinuity in the transmission characteristics. This is done in planar topology by changing the width of the transmission line. Here choose the dimensions of the stubs connecting to the circles as fixed 0.1 $\mathrm{mm}$ in width and $0.15 \mathrm{~mm}$ in length. These circles have different impedances and which can be changed by adjusting the radius of the circles. After adjusting the radius of the circles of the stubs, the resonant modes of the MMR can be roughly designated within the 3.1-10.6 GHz UWB band while demolishing the spurious harmonics in the upper-stop band.

The length can be reduced and the compactness successively increased by introducing inter digital filters, comb-line filters, and parallel-coupled line filters. In order to enhance the coupling degree, two inter digital coupled-lines are used. Conventional inter digital coupled line which has been widely used as a capacitive coupling element in multi-stage band pass filters. The optimized inter digital coupled lines is used to achieve design-specified coupling factor between two adjacent line resonators. For this, the common procedure is to decrease both strip and slot widths in order to achieve a lower insertion and tight coupling. But, it introduces some difficulties into the design and fabrication process because the coupling response is susceptible to the strip or slot 
widths of the configuration. In this paper, the optimized values of strip and slot width are chosen as $0.1 \mathrm{~mm}$ and $0.05 \mathrm{~mm}$ respectively. Coupling length denoted as L should be optimized to enhance the coupling degree.

A new topology of MMR is utilized to replace the traditional MMR. It is formed by attaching three pairs of circular impedance-stepped stubs in shunt to a simple high impedance micro strip line.

\section{OPTIMISATION OF FILTER}

This structure of compact UWB band pass filter is implemented with optimized values of $R_{1}, R_{2}$ and coupling length L. The optimization is completed as follows .Implement the structure with weak coupling, that is consider $L$ as $.6 \mathrm{~mm}$. Change the values of $R_{1}$ by keeping $R_{2}$ as constant and $\mathrm{L}$ as $.6 \mathrm{~mm}$. Check the graphical result of $S_{21}$ for an ultra wide band, high insertion loss in the stop band and wide upper stop band. Allocate the resonating modes in the ultra wide band for a pass band in UWB by keeping $R_{2}$ constant and $R_{1}$ varying. Repeat the above steps for $R_{1}$ constant and $R_{2}$ varying with same weak coupling $L$ as $.6 \mathrm{~mm}$. Finally optimize the values of $R_{1}$ and $R_{2}$ for a pass band in UWB and wide upper stop band. For enhancing the coupling, increase the $\mathrm{L}$ value. This is for the proper feeding of MMR by inter-digital coupling line. Optimize the coupling length for a $0 \mathrm{~dB}$ insertion loss in the pass band. The optimisation can be completed as follows.

1. Implement the structure with weak coupling, that is consider $\mathrm{L}$ as $.6 \mathrm{~mm}$

2. Change the values of $R_{1}$ by keeping $R_{2}$ as constant and $L$ as $.6 \mathrm{~mm}$.

3. Check the graphical result of $S_{21}$ for an ultra wide band, high insertion loss in the stop band and wide upper stop band.

4. Allocate the resonating modes in the ultra wide band for a pass band in UWB by keeping $\mathrm{R}_{2}$ constant and $\mathrm{R}_{1}$ varying.

5. Repeat the above steps for constant $R_{1}$ and varying $R_{2}$ with same weak coupling $L$ as $.6 \mathrm{~mm}$. 6. Finally optimize the values of $R_{1}$ and $R_{2}$ for a pass band in UWB and wide upper stop band.

7. For enhancing the coupling, increase the $L$ value. This is for the proper feeding of MMR by inter digital coupling line.

8. Optimize the coupling length for a $0 \mathrm{~dB}$ insertion loss in the pass band. 


\subsection{EFFECT OF CHANGING $R_{1}$, FIXED $R_{2}=.5 \mathrm{MM}$}
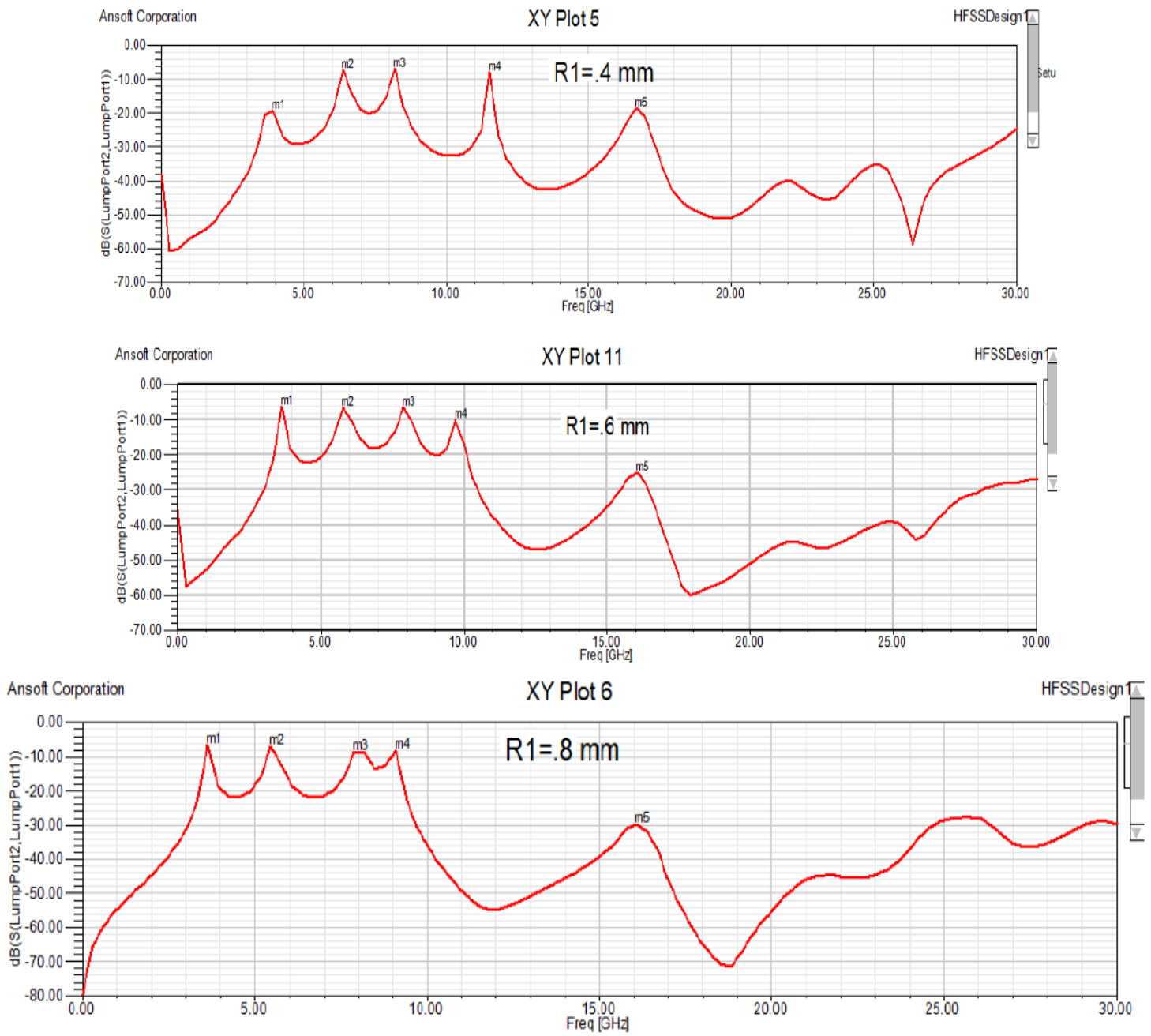

Figure 2: Simulated $S_{21}$-magnitude of the filter under weak coupling with fixed $L=0.6 \mathrm{~mm}, \mathrm{R}_{2}=0.5 \mathrm{~mm}$ and varied $\mathrm{R}_{1}$

As radius varies from 0.4 to $0.8 \mathrm{~mm}$, the odd resonant $(\mathrm{m} 1, \mathrm{~m} 3, \mathrm{~m} 5)$ remain stationary, while the even resonant $(\mathrm{m} 2, \mathrm{~m} 4)$ modes tend to slowly shift downwards. It is well valid in theory that the center location of the resonator corresponds to a short circuit or perfect electrical wall for odd modes, and its characteristics are hardly affected by the attachment of the shunt stub, whereas it indicates an open circuit or perfect magnetic wall for all the even resonant modes. Thus, the second mode can be adjusted to the middle of the pass band, while the fourth mode can be reduced and allocated within the UWB pass band. 
International Journal on Cybernetics \& Informatics (IJCI) Vol. 5, No. 2, April 2016

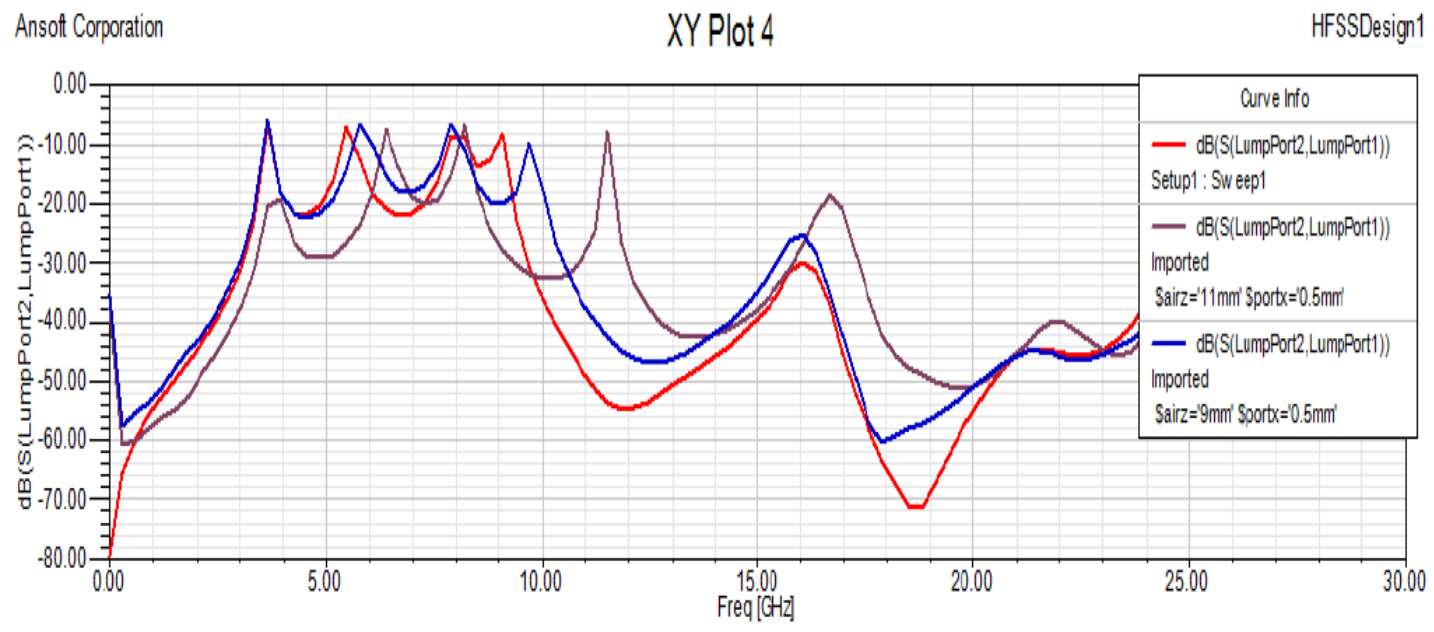

Figure 3: Comparison of $S_{21}$ for different values of $R_{1}$.

\subsection{EFFECT OF CHANGING $R_{2}$, FIXED $R_{1}=.6 \mathrm{MM}$}

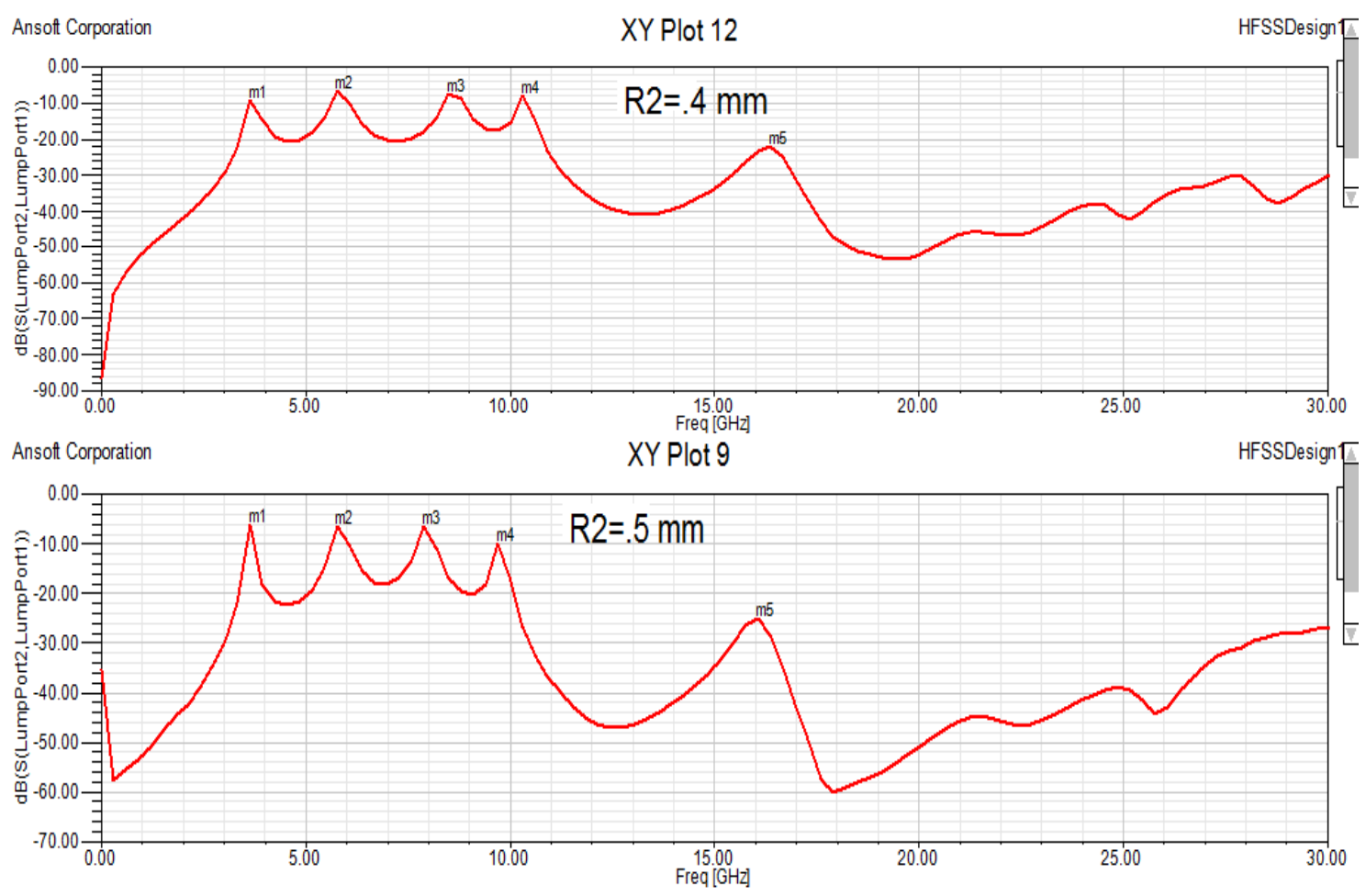


International Journal on Cybernetics \& Informatics (IJCI) Vol. 5, No. 2, April 2016

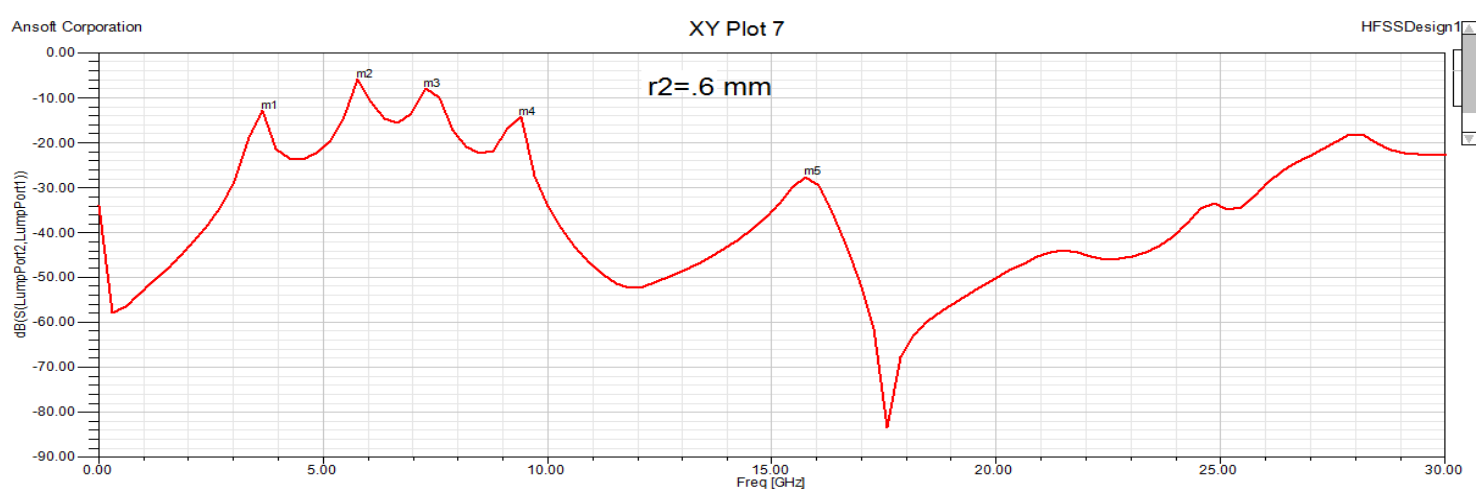

Figure4: Simulated $S_{21}$-magnitude of the filter under weak coupling with fixed $L=0.6, R_{1}=0.6$ and varied $R_{2}$

The five resonant modes all move towards the lower frequency except the second mode which is almost unchanged, while changing the radius $\mathrm{R}_{2}$ from $.4 \mathrm{~mm}$ to $.6 \mathrm{~mm}$. Thus, the two side circular impedance-stepped stubs with varied can provide an additional degree of freedom to adjust the locations of the first four resonant frequencies in an alternative way.

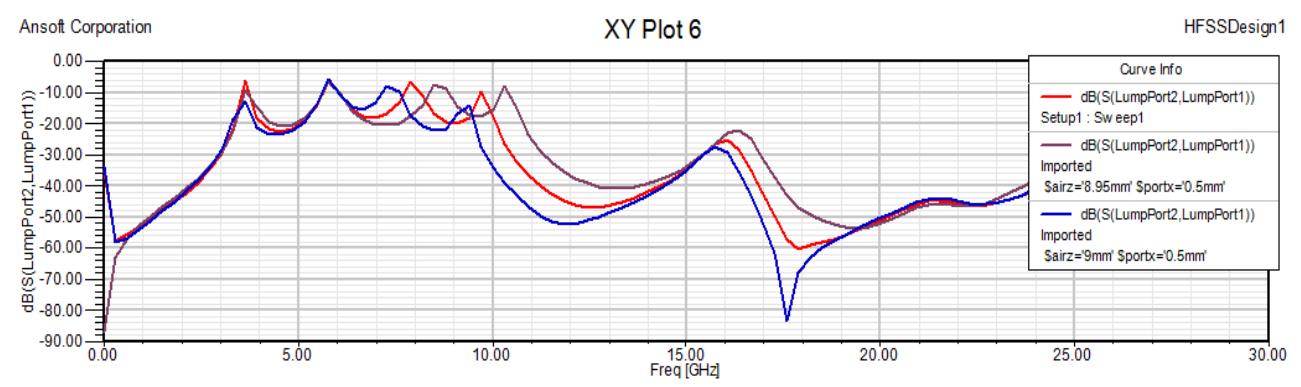

Figure 5: Comparison of $S_{21}$ for different values of $R_{2}$.

\subsection{EFFECT OF CHANGING L, FIXED $R_{1}=.6 \mathrm{MM}$ AND $\mathrm{R}_{2}=.5 \mathrm{MM}$}

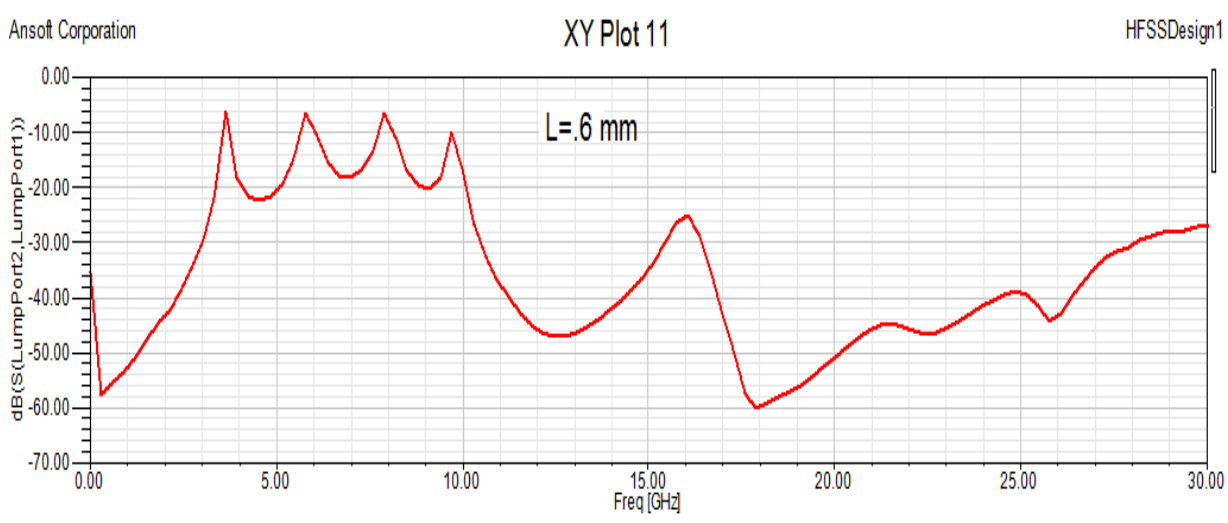


International Journal on Cybernetics \& Informatics (IJCI) Vol. 5, No. 2, April 2016
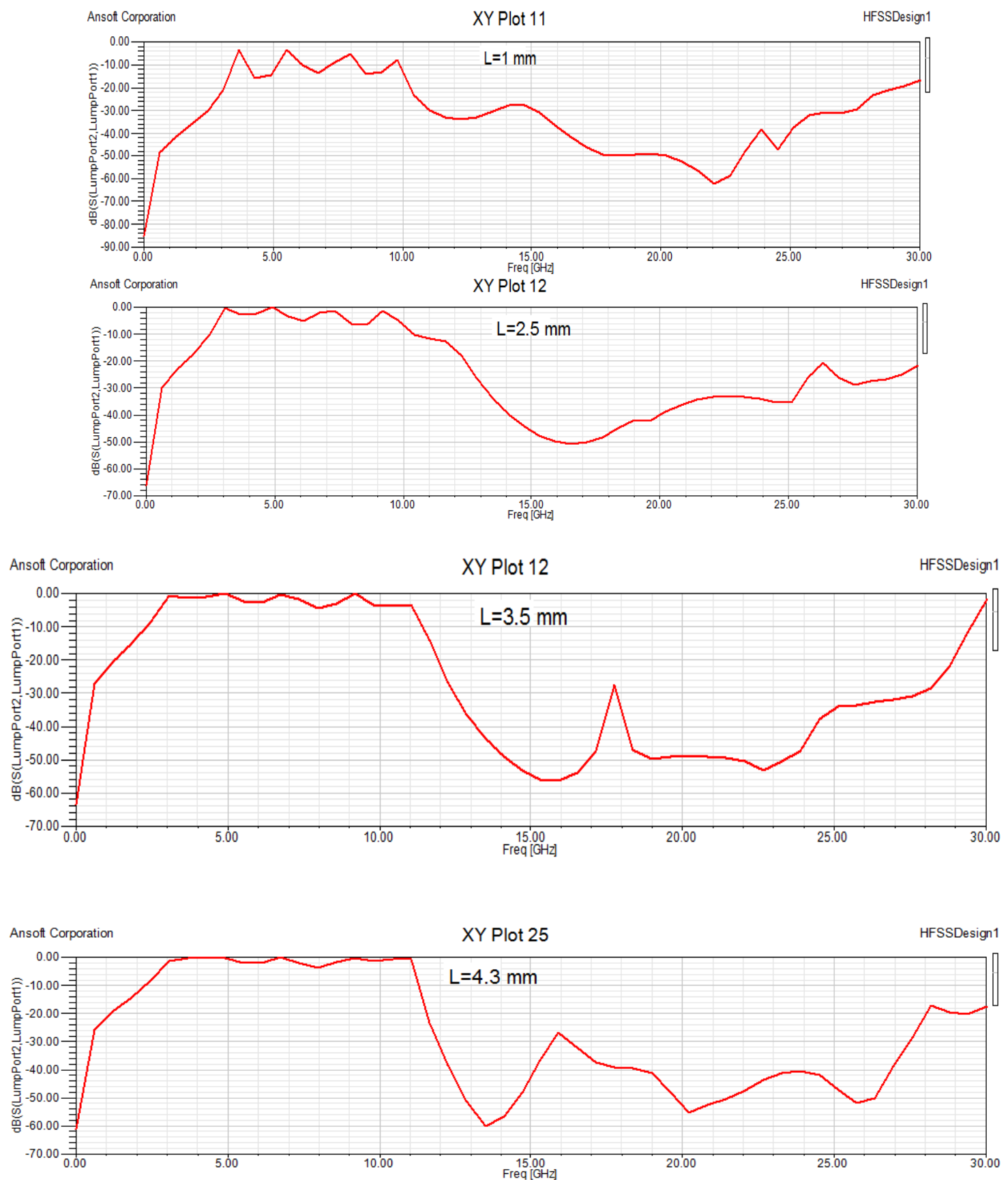

Figure6: Simulated $S_{21}$-magnitude of the filter under weak coupling with varied $L$ and fixed $R_{1}=0.6$ and $\mathrm{R}_{2}=.5 \mathrm{~mm}$

From the above figures, it can be seen that on increasing L improved pass band performance can be achieved. From all these optimisation methods it can be seen that upper stop band performance of the proposed filter is improved. 


\section{RESULTS AND DISCUSSIONS}

\subsection{EQUIVALENT CIRCUIT OF UWB MICROWAVE BAND PASS FILTER AND ANALYSIS}

By Richard transformation and kuroda's identity the schematic of the equivalent circuit of a UWB planar microwave band pass filter. The circuit parameters have the following values

$\mathrm{C}_{1}=\mathrm{C}_{3}=.267 \mathrm{pF}$

$\mathrm{L}_{1}=\mathrm{L}_{3}=2.887 \mathrm{nH}$

$\mathrm{C}_{2}=.7488 \mathrm{pF}$ and

$\mathrm{L}_{2}=1.03 \mathrm{nH}$

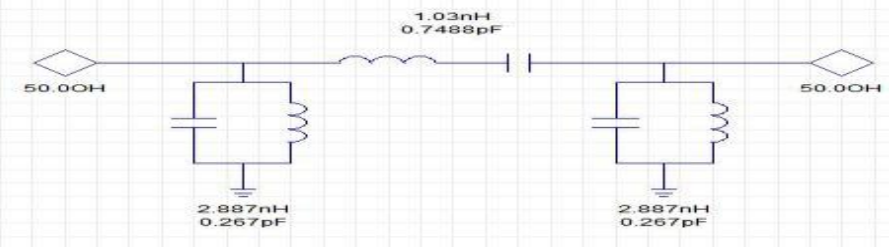

Figure 7: Equivalent circuit

On simulation in HFSS circuit design software $S_{21}$ and $S_{11}$ were obtained as in figure 8.

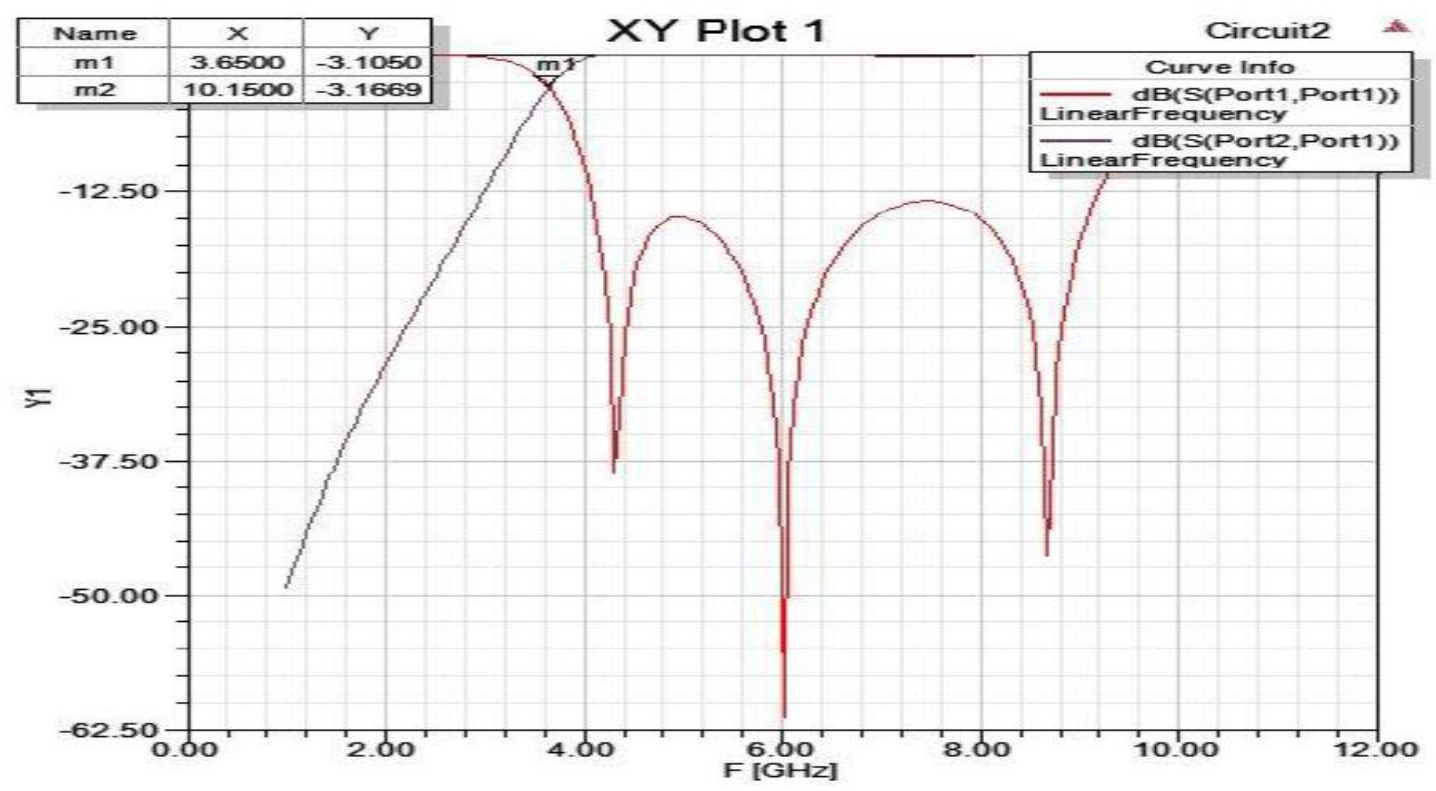

Figure 8: Simulation results 
International Journal on Cybernetics \& Informatics (IJCI) Vol. 5, No. 2, April 2016

\section{FEATURES OF THE DESIGN}

1) Small insertion loss in the pass band.

2) The wide and deep upper-stop band with an insertion loss of $30 \mathrm{~dB}$ in the 12.1 to $27.8 \mathrm{GHz}$.

3) Compact size with $13.6 \mathrm{~mm}$ in length.

\section{CONCLUSION}

In this work, a compact UWB BPF with improved upper stop band performance is proposed and demonstrated using the new MMR which is formed by uniting three circular impedance-stepped stubs in shunt to a high impedance micro-strip line. The design procedure of this circular MMR is much simpler compared to the rectangular MMR. Just by simply adjusting the radius of stubs, the first four resonant modes of this MMR can be successfully allocated within the regulated UWB pass band, which makes the 3-dB bandwidth from 2.8 to $11 \mathrm{GHz}$. Meanwhile, a wide upper-stop band with the insertion loss higher than $30 \mathrm{~dB}$ in range of 12.1 to $27.8 \mathrm{GHz}$ is achieved. In addition, it has a compact size with $13.6 \mathrm{~mm}$ in length.

\section{ACKNOWLEDGEMENT}

I express my deep and profound feeling of gratitude to Mr. Harikrishnan A I, Assistant Professor, NSS College of Engineering, Palakkad for providing me with the guidance for the successful completion of the project

\section{REFERENCES}

[1] L. Zhu, H. Bu, and $\mathrm{K}$. Wu, "Aperture compensation technique for innovative design of ultrabroadband micro strip band pass filter," in IEEE MTT-S Int. Dig., vol. 1, 2000, pp. 315-318.

[2] W. Menzel, L. Zhu, K. Wu, and F. Bogelsack, "On the design of novel compact broad-band planar filters,” IEEE Trans. Microw. Theory Tech., vol. 51, no. 2, pp. 364-370, Feb. 2003.

[3] L. Zhu, W. Menzel, K. Wu, and F. Boegelsack, "Theoretical characterization and experimental verification of a novel compact broadband micro strip band pass filter," in Proc. Asia-Pacific Microwave Conf., Dec.2001, pp. 625-628.

[4] M. Makimoto and S. Yamashita, "Band pass filters using parallel coupled strip line stepped impedance resonators," IEEE Trans. Microw. Theory Tech., vol. 28, no. 12, pp. 1413-1417, Dec. 2001.

[5] S. W. Wong and L. Zhu, "EBG-embedded multiple-mode resonator for UWB band pass filter with improved upper-stopband performance," IEEE Microw.Wireless Compon.Lett., vol. 17, no. 6, pp. 421-423, Jun. 2007.

[6] Ishida, H., and Araki, K.: 'Design and analysis of UWB bandpass filterwith ring filter,' IEEE MTT-S Int. Microw. Symp. Dig., June 2004,pp. 1307-1310.

[7] Ji, M.Z., and Chu, Q.X.: 'A compact UWB band pass filter usingpseudo-interdigital stepped impedance resonators'. Proc. ChinaMicrowave Millimetre-Wave Conf., Ningbo, China, October 2007,pp. 1096-1098.

[8] Zhu, L., and Wang, H.: 'Ultra-wideband bandpass filter on aperturebacked microstrip line,' Electron. Lett., 2005, 41, (18), pp. 1015-1016. 


\section{AUTHOR}

Ambily $\mathrm{K}$ did her B. tech in Electronics and Communication from Calicut university at NSS College of Engineering in 2014.Currently she is pursuing her M. tech in VLSI and Embedded systems at Mar Athanasius College of Engineering, Kothamangalam

Anila P V working as Assistant Professor in Mar Athanasius College of Engineering, Kothamangalam received her M.Tech degree in Electronics with specialization of Microwave and Radar Engineering from Cochin University of Science and Technology (CUSAT) in 2011 and B.Tech in Electronics and Communication Engineering in 2009. She is also pursuing her Ph.D degree from CUSAT. Her areas of interest include Electronic Circuits, Computational Electromagnetics, Multi band antennas, Planar antennas, Metamaterials and its applications in the field of compact

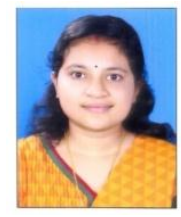
antennas etc

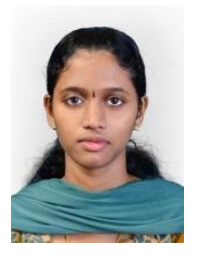

